\title{
Количественный анализ многозональных космических изображений городских агломераций. Часть 2
}

\author{
В.А.Малинников, МИИГАиК, Москва \\ Чан Тиен Ранг, МИИГАиК, Москва
}

Мультифрактальный анализ. Космические изображения земной поверхности представляют собой дискретные множества чрезвычайно высокой размерности. Они характеризуются своей высокой ковариантностью, что является одной из наиболее трудных проблем при обработке дДЗ. Именно из-за неё традиционные подходы к сегментации и классификации космических изображений зачастую являются неэффективными.

Идея применения мультифрактального подхода к обработке высококонтрастных космических изображений основана на присущем им свойстве масштабной инвариантности. Основой этого подхода является выделение из снимка некоторых фундаментальных компонент - сингулярных многообразий. Эти компоненты обладают одинаковым показателем «интенсивности меры» и корректно учитывают локальные структурные свойства различных участков изображения. Сингулярные многообразия позволяют сохранить детальность и уменьшить вариации контраста, что позволяет решать задачи классификации и распознавания образов для таких данных [2].

В настоящем исследовании нами был использован метод обобщённого локально-глобального мультифрактального анализа цифровых изображений, предложенный в работе [3], позволяющий, получать мультифрактальные спектры $\tau(q), D(q)$, $F(\alpha)$ и оценивать локальное поведение меры. Процедура расчёта значений локальных и глобальных мультифрактальных характеристик выполнена в программе Гео-ПК [3]. Процедура расчёта значений локальных и глобальных мультифрактальных характеристик включает в себя следующие этапы:

1) формирование меры ячеек, надстраиваемых над каждым элементом цифрового изображения;

2) расчёт для каждой точки изображения значений гельдеровских показателей $\alpha(x, y)$, характеризующих локальное поведение меры;

3) расчёт значений одномерных мультифрактальных спектров скейлинговых показателей $\tau(q)$, спектров Реньи $D(q)$ и спектров фрактальных размерностей $f_{\mathrm{L}}(\alpha)$, характеризующих глобальное поведение меры;

Расчёт значений локального спектра фрактальных размерностей $f_{L}(\alpha(x, y))$, демонстрирующих, к какому из фрактальных подмножеств принадлежит каждый элемент изображения. Формулы расчёта локальных и глобальных мультифрактальных характеристик приведены в работе [4].

Первая часть статьи «Количественный анализ многозональных космических изображений городских агломераций» опубликована в № 2 журнала «Academia. Архитектура и строительство» за 2019 год.
Спектры показателей массы $\tau(q)$ представляют собой монотонно возрастающие функции, характеризующиеся выраженной кривизной, что является диагностическим признаком мультифрактальности и указывает на существенную гетерогенность описываемых пространственных структур. Спектры обобщённых размерностей Реньи $D(q)$ имеют вид невозрастающих функций от порядка момента с выраженными верхней и нижней асимптотами (см. рис. 2).

На рисунках 2-3 спектры $\tau(q)$ и $D(q)$, рассчитанные для многозональных изображений мегаполиса Ханой, в основном имеют соответствующий мультифрактальной теории канонический вид, однако для зональных изображений, полученных в спектральных каналах: В1 (0,43-0,45 мкм), В2 (0,52-0,60 мкм), B10 (10,60-11,19 мкм) и В11 (11,50-12,51 мкм) данные спектры вырождаются в линейные функции, что указывает либо на отсутствие мультифрактальности, либо на слабую контрастность данных зональных изображений. Визуальный анализ исходных зональных изображений Landsat-8 мегаполиса Ханой показывает, что именно указанные выше спектральные каналы съёмки обладают слабым контрастом, что и обуславливает вырождение (стягивание в точку) мультифрактальных спектров соответствующих зональных изображений мегаполиса.

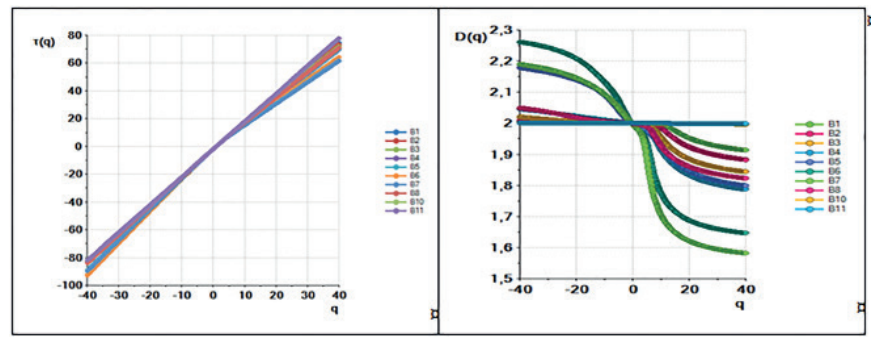

Puc. 2. Массовый спектр $\tau(q)$ и спектр Реньи D(q) многозональных изображений мегаполиса

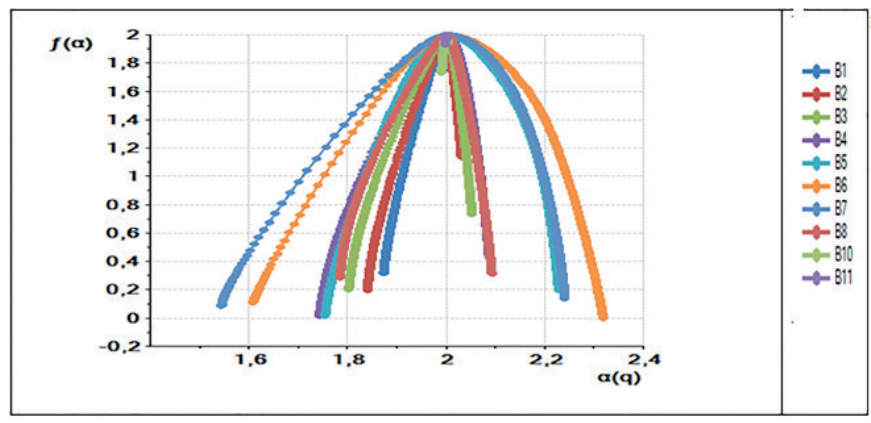

Рис. 3. Мультифрактальные спектры $f(\alpha)$ многозональных изображений мегаполиса 
В силу преобразований Лежандра графики функций размерностей Реньи $D_{\text {q }}$ (см. рис. 2) и мультифрактальный спектр $f(\alpha)$ (см. рис. 3) мегаполиса Ханой сопоставимы между собой. Неотрицательная функция спектра сингулярностей $f(\alpha)$ имеет максимальное значение в точке $\alpha=0=\alpha_{0}$ и в этой точке перегиба графика $f\left(\alpha_{0}\right)=D_{0}$ совпадает с размерностью носителя мультифрактального множества. Правая ветвь мультифрактального спектра (см. рис. 3) соответствует отрицательным значениям q. В этой области графиков размерностей Реньи и мультифрактальных спектров мегаполисов Ханой и Москва фиксируется значительное информационное различие. Вид правой части мультифрактального спектра определяется тем, что при больших отрицательных значениях $q$ подавляющий вклад в статистическую сумму $Z(q, \varepsilon)$ вносят ячейки с малой относительной численностью пикселей $p$. Применительно к рассматриваемому примеру это означает, что изображения пространственной структуры мегаполиса в различных зонах спектра электромагнитных волн разнятся по степени неоднородности. Левая восходящая ветвь спектра характеризует группировку доминирующих элементов уроболандшафта: чем ближе крайняя левая точка спектра к началу координат, тем более выражено доминирование в рассматриваемом множестве $L$.

Анализ значений спектров $f(\alpha)$, представленных на рисунке 3, показал, что обе ветви спектров расширяются вниз и выходят за пределы интервала, имеющего ширину $-1<Q<+1$, что также является признаком наличия у зональных изображений мультифрактальных свойств. У нефракталов обе части спектра располагаются внутри указанного интервала.

Для проведения анализа различий мультифрактальных спектров зональных космических изображений мегаполиса мы воспользовались показателем разброса мультифрактальных свойств исследуемых изображений: $\Delta \alpha=\alpha_{\max }-\alpha_{\min }$ и информационным параметром $\Delta D=D_{1}-D_{\infty}$. Величина $\Delta D$ численно совпадает с разбросом значений спектра $D_{q}$, а величина $\Delta \alpha-$ с шириной спектра $f(\alpha)$. Величина $\Delta \alpha$ характеризует «контрастность» вероятностей заполнения структурных ячеек. Чем больше $\Delta \alpha$, тем больший разброс фрактальных свойств демонстрирует исследуемое изображение. Величина $\Delta D=D_{1}-D_{\alpha^{\prime}}$ или её оценка $\Delta_{\mathrm{Q}} D=D_{1}-D_{\mathrm{Q}}$ (где $Q-$ некоторое положительное достаточно большое значение параметра $q$, задаваемое в конкретных вычислениях), занимает центральное место в информационной интерпретации мультифрактального формализма. Увеличение $\Delta_{\mathrm{Q}} D$ для исследуемой серии зональных изображений мегаполиса показывает, что в структуре становится больше периодической составляющей, и что система накачивается информацией (негэнтропией) и в ней возрастает степень нарушения симметрии. Таким образом, показатель отражает степень упорядоченности и нарушения симметрии общей конфигурации структуры земной поверхности. Большие значения величины $\Delta_{Q} D$ соответствуют большей степени нарушения симметрии. Оба количественных критерия могут использоваться наравне. Однако на практике использование параметра $\Delta \alpha$ в ряде случаев оказывается более предпочтительным, поскольку спектр $D(q)$ убывает гораздо медленнее спектра $\alpha(q)$ и, как следствие, для адекватной оценки величины $\Delta_{Q} D$ требуется расчёт моментов высоких порядков. Для всех анализируемых нами изображений мегаполисов были вычислены значения параметра аспекта $r$ и коэффициента асимметрии $R$ для $f(\alpha)$ спектров, характеризующих пространственную структуру зональных изображений мегаполиса [4].

В таблице 1 представлен перечень наиболее информативных мультифрактальных параметров, которые использовались нами для дифференциации исследуемых изображений городских агломераций.

Коэффициент $R$ принимает значения из диапазона $[-1$, 1] и характеризует направление и степень скошенности спектра $f(\alpha)$. Если $R>0$, то спектр $f(\alpha)$ скошен влево, а при $R<0$ спектр $f(\alpha)$ скошен вправо. Спектр $f(\alpha)$ симметричен, если $R$ близко к нулю.

Рассчитанные характеристики мультифрактальных спектров зональных изображений мегаполиса Ханой представ-

Таблица 1. Перечень наиболее информативных мультифрактальных параметров

\begin{tabular}{|c|c|}
\hline 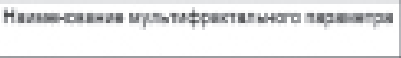 & 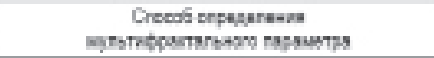 \\
\hline 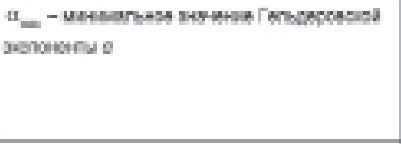 & 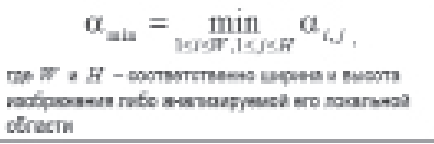 \\
\hline 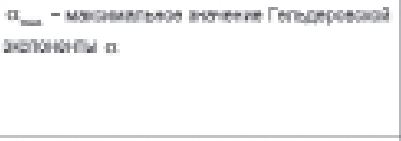 & 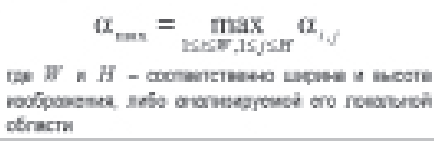 \\
\hline 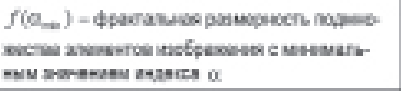 & $f\left(\alpha_{\sin }\right)=\inf _{\mathcal{A} \rightarrow A}\left\{\alpha_{\operatorname{sis}} q-\tau(q)\right\}$ \\
\hline 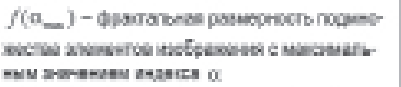 & $f\left(\alpha_{\cos }\right)=\inf _{i<A}\left\{\alpha_{\operatorname{cox}} q-\tau(q)\right\}$ \\
\hline 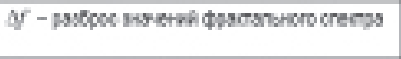 & $\Delta f=f\left(\alpha_{\max }\right)-f\left(\alpha_{\mathrm{mb}}\right)$ \\
\hline 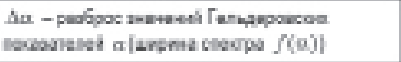 & $\Delta \alpha=\alpha_{\min }-\alpha_{\min }$ \\
\hline 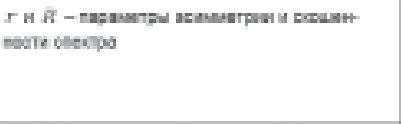 & 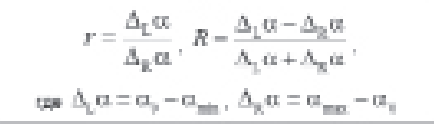 \\
\hline 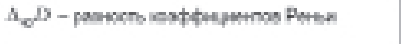 & $\Delta_{*} D=D_{1}-D_{*}$ \\
\hline
\end{tabular}

Таблица 2. Характеристики мультифрактальных спектров зональных изображений Landsat мегаполиса Ханой

\begin{tabular}{|c|c|c|c|c|c|c|c|c|c|c|c|}
\hline & $\Delta 4$ (мm) & $D_{0}$ & $D_{i}$ & $D_{2}$ & $D_{40}$ & $\Delta u D$ & $r$ & $R$ & $a_{\max }$ & $a_{\min }$ & $\Delta a$ \\
\hline B1 & $0,433-0,453$ & 2 & 2 & 2 & 1,914 & 0,086 & 8,84 & 0,8 & 2,01 & 1,87 & 0,14 \\
\hline B2 & $0,450-0,515$ & 2 & 2 & 2 & 1,882 & 0,118 & 3,6 & 0,56 & 2,03 & 1,84 & 0,19 \\
\hline B3 & $0,525-0,600$ & 2 & 2 & 2 & 1,844 & 0,156 & 3,13 & 0,52 & 2,05 & 1,8 & 0,25 \\
\hline B4 & $0,630-0,680$ & 2 & 1,998 & 1,996 & 1,788 & 0,210 & 2,5 & 0,43 & 2,09 & 1,74 & 0,35 \\
\hline B5 & $0,845-0,885$ & 2 & 1,993 & 1,988 & 1,799 & 0,204 & 0,88 & 0,06 & 2,23 & 1,75 & 0,48 \\
\hline B6 & $1,560-1,660$ & 2 & 1,987 & 1,979 & 1,647 & 0,34 & 1,07 & 0,03 & 2,32 & 1,61 & 0,71 \\
\hline B7 & $2,100-2,300$ & 2 & 1,987 & 1,977 & 1,582 & 0,405 & 1,55 & 0,21 & 2,24 & 1,54 & 0,7 \\
\hline B8 & $0,500-0,680$ & 2 & 2 & 2 & 1,823 & 0,177 & 2,4 & 0,41 & 2,09 & 1,78 & 0,31 \\
\hline B10 & $10,30-11,30$ & 2 & 2 & 2 & 1,997 & 0,003 & 3,34 & 0,54 & 2,01 & 1,99 & 0,02 \\
\hline B11 & $11,50-12,50$ & 2 & 2 & 2 & 2 & 0 & 1,94 & 0,32 & 2 & 1,99 & 0,01 \\
\hline
\end{tabular}




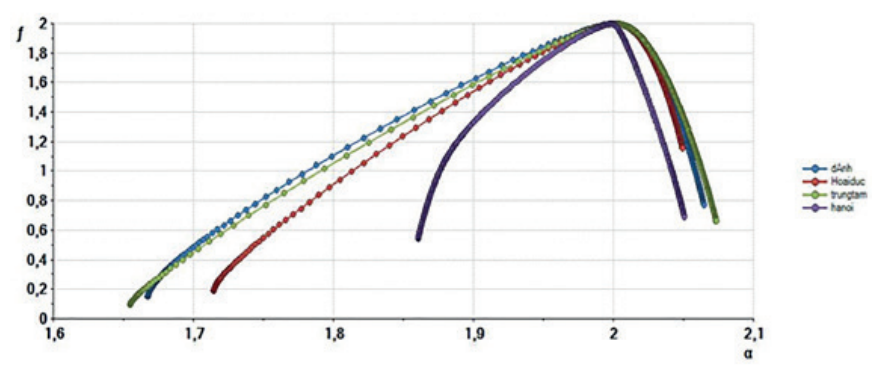

Рис. 4. Мультифрактальные спектры отдельных городских агломераций и в целом мегаполиса Ханой

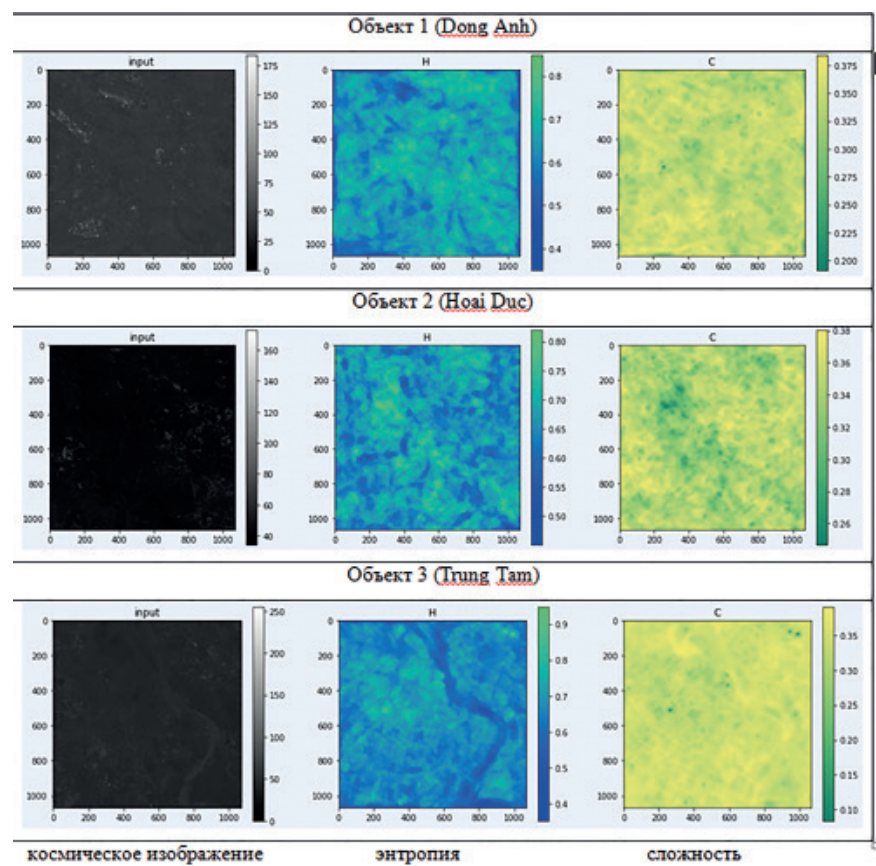

Puс. 5. Распределение энтропии и сложности на космическом изображении

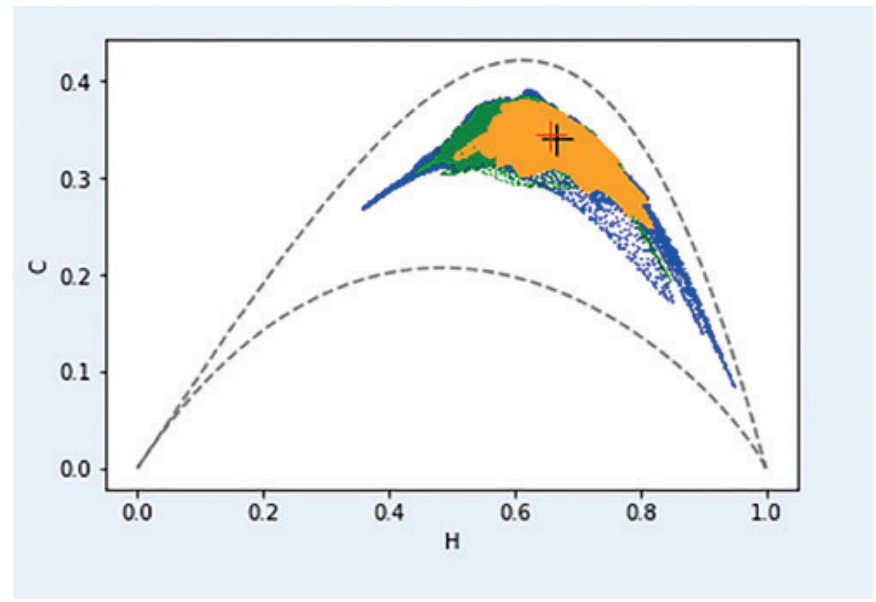

Рис. 6. Диаграмма энтропийных показателей, характеризующая изменение степени упорядоченности пространственной ориентации элементов в системе координат «энтропия - сложность» по изображению городской агломерации. Крестик - среднее значение «энтропия - сложность» для данной городской агломерации лены в таблице 2. Анализ изменчивости значений данных характеристик в зависимости от спектрального диапазона съёмки показывает, что статистически значимо отличаются для различных зональных изображений мегаполиса Ханой такие параметры как: $\Delta \alpha=\alpha_{\max }-\alpha_{\min }, \Delta_{40} D=D_{1}-D_{40}, r$ и $R$.

C целью изучения неоднородности пространственной структуры мегаполиса Ханой мы рассчитали значения мультифрактальных спектров $D_{q}$ и $f\left({ }_{\alpha}\right)$ для трёх фрагментов панхроматического изображения мегаполиса Ханой.

Представленные на рисунке 4 мультифрактальные спектры демонстрируют существенную изменчивость мультифрактальных характеристик для различных городских агломераций мегаполиса Ханой, указывающую на неоднородность пространственной структуры мегаполиса, которая может быть количественно описана с помощью вышеупомянутых характеристик мультифрактальных спектров.

\section{Метод диаграмм «сложность-энтропия»}

При проведении исследования степени упорядоченности пространственной структуры городской агломерации использовались их космические изображения, полученные со спутника Ландсат. Исследования изображений позволили оценить степень упорядоченности пространственной ориентации элементов городской агломерации в системе координат «энтропия-сложность». Была использована программа определения статистической сложности на основе расхождения Йенсена-Шеннона между наблюдаемым и равновероятным распределением коэффициентов разложения, позволяющая оценить уровень нетривиальности наблюдаемого пространственного паттерна, например, отнести его к реализации полностью случайного процесса, детерминированного хаоса или регулярной динамики [5].

Расчёты проводились с использованием стандартных библиотек языка Python для научных расчётов и визуализации, библиотеки для шеарлет-разложения в PyShearlets, а также программного обеспечения на языке Python [6]. На рисунке 5 показаны распределения энтропии и сложности на снимке городской агломерации в зависимости от места нахождения точки на изображении, рядом приведена цветовая шкала величины изменения параметра в относительных единицах (с нормировкой на максимально возможное значение).

Разброс значений энтропии, определённой по всему изображению (рис. 6) достаточно велик: от 0,4 до 0,9 отн. ед., для сложности от 0,1 до 0,38 отн. ед. В рамках программы пространственный паттерн пространственной структуры городской агломерации предлагается описывать как точку на плоскости в координатах «энтропия-сложность», где нулевая энтропия и сложность соответствуют полностью регулярной структуре, а высокая энтропия и нулевая сложность соответствуют полностью случайному пространственно независимому шуму [7]. Соответственно в качестве характеристики городской агломерации принималось среднее значение «энтропия-сложность» для данного изображения - красная точка на графике (рис. 6), где зелёным цветом показана вся 
область изменения параметров «энтропия - сложность» для данной городской агломерации.

На диаграммах «энтропия-сложность»:

- синим цветом и фиолетовым крестиком показаны результаты для изображения Trungtam;

- зелёным цветом и красным крестиком показаны результаты для изображения dAnhsSon;

- оранжевым цветом и чёрным крестиком показаны результаты для изображения HoaiDuc.

На изображениях различных городских агломераций встречаются как похожие на первый взгляд, так и отличающиеся участки. Полученные средние значения «энтропиясложность» для каждого изображения, позволяют провести анализ этих значений для любых исследуемых участков. Так, можно показать, что участки территории, характеризующиеся неупорядоченной пространственной структурой, заметно отличаются по значениям «энтропия-сложность». Обычно слабо организованная пространственная структура городской агломерации состоит из перемежающихся сравнительно упорядоченных и неупорядоченных областей, и её пространственные точки располагаются в двух зонах: минимального значения сложности и максимальной энтропии и в области максимальной сложности со значениями энтропии 0,6-0,8.

Таким образом, оценка пространственной сложности и энтропии для космических изображений городских агломераций, а также статистические характеристики значений «энтропия-сложность» позволяют различать упорядоченные и неупорядоченные городские агломерации. Причём наиболее достоверно эти показатели позволяют диагностировать наличие высокоорганизованной агломерации в мегаполисе.

\section{Литература}

1. Чан Жанг Нам. Трансформация объёмно-планировочной структуры Ханоя в первой половине XX века / Чан Жанг Нам // Вестник гражданских инженеров. - 2016. - № 3 (56). - С. 40-45.

2. Макаренко, Н.Г. Скейлинговые свойства цифровых изображений земных ландшафтов / Н.Г. Макаренко, Л.М. Каримова, О.А Круглун // Современные проблемы дистанционного зондирования земли из космоса. - 2014. - Т 11. - № 2. - С. 26-37.

3. Учаев, Дм. В. Разработка метода обобщённого локальноглобального мультифрактального анализа изображений для исследования пространственной структуры сложных природно-антропогенных систем / Дм.В. Учаев, В.А. Малинников, Д.В. Учаев // Извести высших учебных заведений «Геодезия и аэрофотосъемка». - 2010. - №4. - С. 64-68.

4. Разведочный мультифрактальный анализ многозональных космических изображений / В.А. Малинников, Д.В. Учаев, Дм.В. Учаев, Ч.Т. Ранг // Известия вузов. Геодезия и аэрофотосъемка. - 2018. - Т. 62. - № 5. - С. 569-581.
5. Intensive entropic non-triviality measure / P.W. Lamberti, M.T Martin, A. Plastino, 0.A. Rosso // Physica A: Statistical Mechanics and its Applications. - Vol. 334, Iss. 1-2, - 2004. - P 119-131. ISSN 0378-4371; https://doi.org/10.1016/j. physa.2003.11.005

6. Brazhe, A. Shearlet-based measures of entropy and complexity for two-dimensional patterns / A. Brazhe // Physical Review E 97. - 2018. - P. 061301 (7).

7. Zunino, L. Discriminating image textures with the multiscale two-dimensional complexity-entropy causality plane / L. Zunino, H.V. Ribeiro // Chaos, Solitons and Fractals. - 2016. - № 91. - P. 679-688.

\section{References}

1. Chan Zhang Nam. Transformatsiya ob"emno-planirovochnoi struktury Khanoya v pervoi polovine XX veka [Transformation of the space-planning structure of Hanoi in the first half of the XX century]. Vestnik grazhdanskikh inzhenerov [Bulletin of Civil Engineer], 2016, no. 3 (56), pp. 40-45.

2. Makarenko N.G., Karimova L.M., Kruglun 0.A. Skeilingovye svoistva tsifrovykh izobrazhenii zemnykhlandshaftov [Scaling properties of digital images of terrestrial landscapes]. Sovremennye problemy distantsionnogo zondirovaniya iz kosmosa [Modern problems of remote sensing of the earth from space], 2014, vol. 11, no.2, pp. 26-37.

3. Uchaev Dm.V., Malinnikov V.A., Uchaev D.V. Razrabotka metoda obobshchennogo lokal'no-global'nogo mul'tifraktal'nogo analiza izobrazhenii dlya issledovaniya prostranstvennoi struktury slozhnykh prirodnoantropogennykh system. Izvestiya vysshikh uchebnykh zavedenii. Geodeziya i aerofotos"emka [Proceedings of the Higher Educational Institutions. Izvestia vuzov "Geodesy and aerophotosurveying"], 2010, no. №4, pp. 64-68.

4. Malinnikov V.A., Uchaev D.V., Uchaev Dm.V., Rang Ch.T. Razvedochnyi mul'tifraktal'nyi analiz mnogozonal'nykh kosmicheskikh izobrazhenii [Exploratory multi-fractal analysis of multispectral space images]. Izvestiya vysshikh uchebnykh zavedenii. Geodeziya i aerofotos"emka [Proceedings of the Higher Educational Institutions. Izvestia vuzov "Geodesy and aerophotosurveying"], 2018, vol. 62, no. 5, pp. 569-581.

5. Lamberti P.W., Martin M.T., Plastino A., Rosso 0.A. Intensive entropic non-triviality measure. Physica $A$ : Statistical Mechanics and its Applications, 2004, Vol. 334, Iss. 1-2, pp. 119-131. ISSN 0378-4371; https://doi.org/10.1016/j. physa.2003.11.005

6. Brazhe A. Shearlet-based measures of entropy and complexity for two-dimensional patterns. Physical Review $E$, 2018, no. 97, pp. 061301(7).

7. Zunino L., Ribeiro H.V. Discriminating image textures with the multiscale two-dimensional complexity-entropy causality plane. Chaos, Solitons and Fractals, 2016, no. 91, pp. 679-688. 
Малинников Василий Александрович (Москва). Доктор технических наук, прфессор. Заведующий кафедрой космического мониторинга и экологии ФГБОУ В0 «Московский государственный университет геодезии и картографии» (105064, Москва, Гороховский пер., 4. МИИГАиК).Эл.почта: malinnikov@mail.ru.

Чан Тиен Ранг (Москва). Аспирант ФГБОУ В0 «Московский государственный университет геодезии и картографии» (105064, Москва, Гороховский пер., 4. МИИГАиК). Эл.почта: malinnikov@mail.ru.

Malinnikov Vasily Alexandrovich (Moscow). Doctor of Technical Sciences, Professor. Head of the Department of Space Monitoring and Ecology of the Moscow State University of Geodesy and Cartography (4 Gorokhovskylane, Moscow, 105064. MIIGAiK). E-mail: malinnikov@mail.ru.

Chang Tien Rang (Moscow). Postgraduate student of the Moscow State University of Geodesy and Cartography (4 Gorokhovsky lane, Moscow, 105064. MIIGAiK).E-mail: malinnikov@mail.ru. 